\title{
Preparation and characterization of graphene oxide aerogels: exploring the limits of supercritical $\mathrm{CO}_{2}$ fabrication methods
}

\author{
Alejandro Borrás, ${ }^{\text {a }}$ Gil Gonçalves, ${ }^{\text {a }}$ Gregorio Marban, ${ }^{\text {b }}$ Stefania Sandoval, ${ }^{\mathrm{a}}$ Susana \\ Pinto, ${ }^{\mathrm{c}}$ Paula A. A. P. Marques, ${ }^{\mathrm{c}}$ Julio Fraile, ${ }^{\mathrm{a}}$ Gerard Tobias, ${ }^{\mathrm{a}}$ Ana M. López-Periago, ${ }^{\text {a }}$ \\ Concepción Domingo ${ }^{* a}$
}

\begin{abstract}
The supercritical carbon dioxide synthesis of non-reduced graphene oxide (GO) aerogels from dispersions of GO in ethanol is here reported as a low-cost, efficient and environmentally friendly process. The preparation is carried out at the mild conditions of $333 \mathrm{~K}$ and $20 \mathrm{MPa}$. The high aspect ratio of the used GO sheets (ca. 30 $\mu \mathrm{m}$ lateral dimensions) allowed the preparation of aerogel monoliths by simultaneous $\mathrm{scCO}_{2}$ gelation and drying. Solid state characterization results indicate that a thermally stable mesoporous non-reduced GO aerogel was obtained by using the supercritical procedure, keeping the GO sheets most of the surface oxygenated groups, thus, facilitating further functionalization. Moreover, the monoliths have a very low density, high specific surface area and excellent mechanical integrity, characteristics which rivals with that of most light-weight reduced graphene aerogels reported in the literature.
\end{abstract}

\section{Introduction}

Graphene is a two-dimensional structure made of carbon atoms with unique electronic, chemical, textural and mechanical properties, but difficult to exfoliate into separate flakes. ${ }^{[1]}$ The most studied route to obtain exfoliated graphene is through the formation of intermediate graphene oxide (GO) followed of subsequent deoxygenation, thus 
leading to reduced graphene oxide (rGO) aerogels. ${ }^{[2]}$ The intermediate GO, synthesized by treatment of graphite with strong mineral acids and oxidizing agents, is a material heavily oxygenated and easily exfoliable. The high stability of GO dispersions in water is the starting key point exploited for the formation of rGO hydrogels by self-assembly under hydrothermal conditions. ${ }^{[3-7]}$ Those hydrogels must be further dried, avoiding the collapse of the porous structure, in order to obtain 3D aerogels with a sponge-like configuration. Opposed to graphene aerogels, the properties of 3D GO structures have been scarcely studied, due to the high chemical instability and poor mechanical properties of these networks. Merely, lyophilization has been applied to the straight drying of GO aqueous dispersions, but obtained GO networks should be further reduced to rGO to get reasonable mechanical stability. ${ }^{[8]}$ Indeed, gelation methods eluding the reduction of GO are difficult to design. Nevertheless, a large number of applications can be envisaged for non-reduced GO aerogels, since they combine the textural advantages of graphene with convenient hydrophilicity and fascinating surface chemistry given by the oxygen functional groups decorating the sp2 basal planes. ${ }^{[9]}$ Certainly, GO aerogels, pristine or as a composite, are appealing materials in electronics, clean energy, pollutants adsorption devices and biomedicine. ${ }^{[10-14]}$

Common drying paths for graphene hydrogels are low-temperature freeze-drying ${ }^{[15]}$ and high-temperature supercritical drying (e.g., at the critical point of short C-chain alcohols). ${ }^{[16,17]}$ Critical point drying techniques are widely used to prepare inorganic aerogels, since it is the best known technique to minimize monolith shrinkage. ${ }^{[18]}$ However, this procedure requires the use of temperatures higher than $525 \mathrm{~K}$, being alcohols dangerously flammable under these conditions. Moreover, this technique removes the remaining oxygen-containing functional groups in the structure of the gel. ${ }^{[19]}$ Opposite to the high-temperature critical point drying process, this work 
describes a low-temperature supercritical carbon dioxide $\left(\mathrm{scCO}_{2}\right)$ method for the production of ultra-low density 3D GO stable macrostructures, integrating the advantages of efficiency, environmental friendless, safety and low-cost. Gelling and drying are performed under mild conditions of pressure and temperature, thus substantial conversion of GO to rGO is prevented. Processes assisted by $\mathrm{scCO}_{2}$ have been previously applied to the drying of pre-formed inorganic and organic alcogels. ${ }^{[12-}$ ${ }^{22]}$ In constructing graphene structures, $\mathrm{scCO}_{2}$ has only recently been proposed as a plausible medium for exfoliation and drying. ${ }^{[23-26]}$ In an step forward, the method developed in this work is devoted to the formulation of stable 3D GO aerogel structures by direct self-assembly of dispersed GO sheets in ethanol in only one step, using exclusively low temperature $\mathrm{scCO}_{2}$ technology and avoiding the use of any crosslinking agent. The developed $\mathrm{scCO}_{2}$ process is optimized for different experimental parameters, such as temperature and $\mathrm{CO}_{2}$ flow. The microstructure, texture, surface chemistry and mechanical properties of synthetized GO aerogels are deeply characterized and compared with data obtained from a sample synthetized with the more conventional hydrothermal/ lyophilization procedure.

\section{Results and Discussion}

Figure 1 shows schematically the main steps involved in the two synthetic procedures contrasted in this work for aerogel formation: the conventional, combining hydrothermal and lyophilization steps, and the newly developed supercritical $\mathrm{CO}_{2}$ method. In the hydrothermal treatment, the final product is obtained straightforwardly from the commercial sol of GO dispersed in water. This suspension has long-term stability due to H-bond and electrostatic repulsion of functional groups in GO. Contrarily, because of the lack of miscibility between $\mathrm{CO}_{2}$ and water, in the 
supercritical method a previous step of water exchange by an organic solvent highly soluble in $\mathrm{scCO}_{2}$ is required. Ethanol was chosen for this purpose.

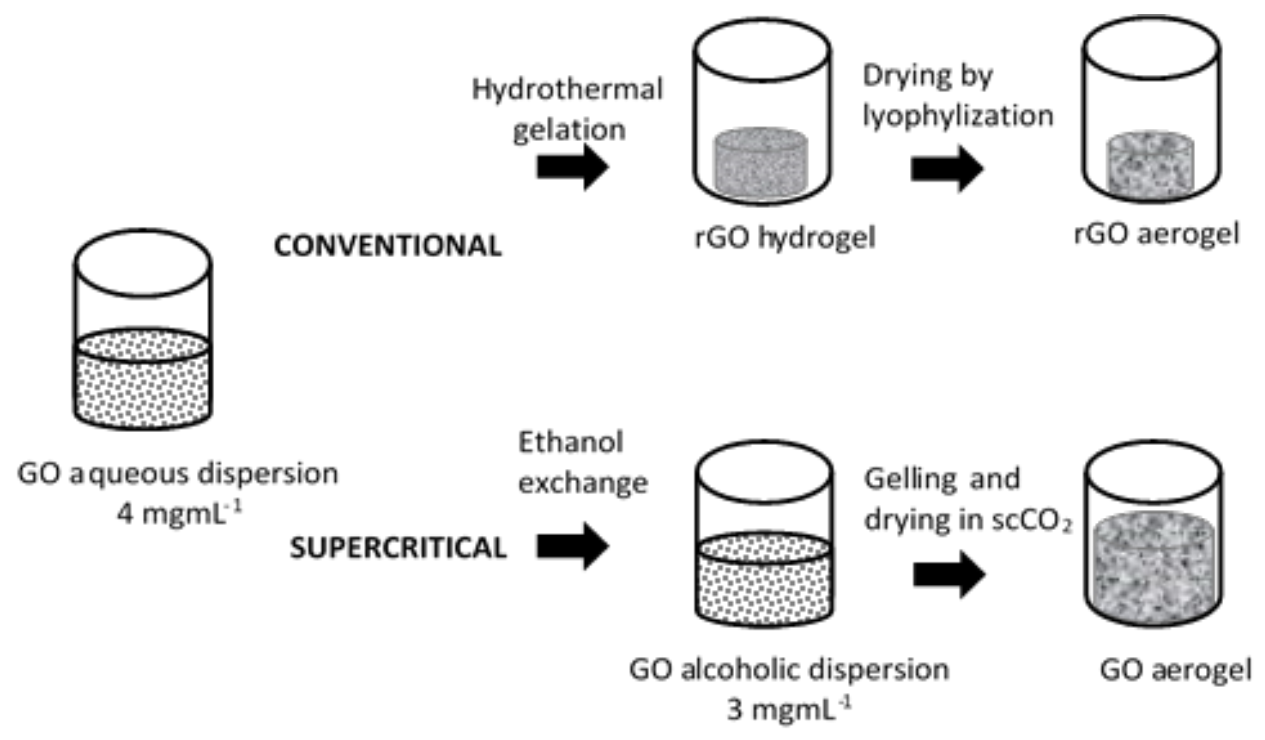

Figure 1. Schematic representation of the main synthetic steps employed in the conventional (hydrothermal / lyophilization) and supercritical $\left(\mathrm{scCO}_{2}\right)$ tested techniques.

It is worth mentioning that ethanol has previously been identified as a non-suitable dispersion medium for GO flakes. ${ }^{[29]}$ A similar result was obtained in this work in preliminary tests performed by straightforwardly interchanging water by ethanol in only one step. In fact, the multi-step water-to-ethanol exchange procedure described in the experimental section was necessary to obtain a long-term stable sol of GO dispersed in ethanol. This multi-step procedure is universally used for solvent exchange in hydrogels to form alcogels. ${ }^{[30]}$

\section{Starting material characterization}

The chemical structure of pristine GO sheets must be known to further understand the aerogel formation mechanism. GO flakes composition is a subject of considerable 
Table 1. XPS measured percentage of carbon in the different functional groups for raw GO, samples upon supercritical and conventional treatment and after thermal annealing.

debate in the literature due to the nonstoichiometric complexity of this material, including batch-to-batch variability. In this work, the chemical composition of asreceived $\mathrm{GO}$ dispersed in water $\left(\mathrm{GOd} \_\mathrm{H}_{2} \mathrm{O}\right)$ and after solvent exchange to ethanol (GOd_EtOH) was elucidated by XPS and ATR infrared characterization techniques. Before measurement, solvents were eliminated by evaporation under vacuum at room temperature.

Table 1 and Figure 2 show the relative abundance of main components in the XPS C 1s spectra of GO flakes $(\mathrm{C}=\mathrm{C}, \mathrm{C}-\mathrm{O}-\mathrm{C}, \mathrm{C}-\mathrm{OH},>\mathrm{C}=\mathrm{O} \& \mathrm{R}-\mathrm{O}-\underline{\mathrm{C}}=\mathrm{O} \& \mathrm{O}=\mathrm{C}-\mathrm{O}-$ $\mathrm{C}=\mathrm{O}, \mathrm{CH}_{\mathrm{x}}$ and $\left.-\underline{\mathrm{C}}-(\mathrm{C}=\mathrm{O})-\mathrm{R}\right)$ for the different raw and processed samples, together with the calculated C/O ratios. XPS analysis of sample $\mathrm{GOd} \_\mathrm{H}_{2} \mathrm{O}$ resulted in a C/O atomic ratio of 2.0, which represents a high degree of oxidation for the flakes. The exchange with ethanol did not produce any noticeable reduction on the sheets, as can be observed for sample GOd_EtOH with a C/O atomic ratio of 2.1. The ethanol reduction effect of GO flakes has only been described for systems at high pressure and temperature, preferentially maintaining the alcohol at supercritical conditions. ${ }^{[31]}$ Deconvolution of the spectra obtained for GOd_H $\mathrm{H}_{2} \mathrm{O}$ and GOd_EtOH indicated the presence of the typical $\mathrm{C}=\mathrm{C} \mathrm{sp}^{2}$ bonds together with hydroxyl and epoxy functionalities in significant percentage, with only a small contribution of ketone and carboxyl functionalities (ca. 5 $\%$ of $>\mathrm{C}=\mathrm{O}$ and $\mathrm{O}=\mathrm{C}-\mathrm{O})$. Carboxylic acid $(-\mathrm{COOH})$ was not detected. 


\begin{tabular}{|c|c|c|c|c|c|c|c|c|}
\hline \multirow{2}{*}{ Specie } & \multirow{2}{*}{$\begin{array}{l}\text { GOd_ } \\
\mathrm{H}_{2} \mathrm{O}\end{array}$} & \multirow{2}{*}{$\begin{array}{l}\text { GOd_ } \\
\text { EtOH }\end{array}$} & \multirow{2}{*}{$\begin{array}{l}\text { GOa_ }_{-} \\
333\end{array}$} & \multirow{2}{*}{$\begin{array}{l}\text { GOa_ }_{-} \\
363\end{array}$} & \multirow{2}{*}{$\begin{array}{l}\text { GOa }_{-} \\
\text {HL }\end{array}$} & \multirow{2}{*}{$\begin{array}{l}\text { GOaT }_{-} \\
393\end{array}$} & \multirow{2}{*}{$\begin{array}{l}\text { GOaT }_{-} \\
573\end{array}$} & \multirow{2}{*}{$\begin{array}{l}\text { GOaT }_{-} \\
1323\end{array}$} \\
\hline & & & & & & & & \\
\hline \% O total & 33.3 & 32.5 & 32.3 & 32.6 & 13.5 & 32.8 & 13.6 & 4.4 \\
\hline \% C total & 65.8 & 67.4 & 67.5 & 67.1 & 86.4 & 67.0 & 85.7 & 95.2 \\
\hline $\mathrm{C} / \mathrm{O}$ & 2.0 & 2.1 & 2.1 & 2.1 & 6.4 & 2.0 & 6.3 & 22 \\
\hline \%Chemisorbed $\mathrm{H}_{2} \mathrm{O}$ & 0.0 & 0.0 & 0.0 & 0.0 & 1.9 & 0.0 & 1.9 & 0.1 \\
\hline$\% \mathrm{C}$ in $\mathrm{C}=\mathrm{C}$ & 28.0 & 27.3 & 26.0 & 27.3 & 52.3 & 24.8 & 45.8 & 44.6 \\
\hline \%C in $\mathrm{CH}_{\mathrm{x}}$ & 0.0 & 0.0 & 0.0 & 0.0 & 0.0 & 0.0 & 0.0 & 24.8 \\
\hline$\% \mathrm{C}$ in $\mathrm{CN}$ & 0.8 & 0.2 & 0.2 & 0.3 & 0.2 & 0.3 & 0.7 & 0.4 \\
\hline$\% \mathrm{C}$ in $\mathrm{C}-\mathrm{O}-\mathrm{C}$ & 9.4 & 13.3 & 20.0 & 15.9 & 6.5 & 20.7 & 0.2 & 4.2 \\
\hline$\% \mathrm{C}$ in $\mathrm{C}-\mathrm{OH}$ & 22.9 & 19.5 & 15.8 & 17.8 & 1.0 & 16.1 & 3.1 & 0.3 \\
\hline$\% \mathrm{C}$ in $>\mathrm{C}=\mathrm{O}$ & 3.7 & 2.9 & 3.5 & 4.7 & 1.8 & 3.3 & 2.7 & 1.1 \\
\hline$\% \mathrm{C}$ in $\mathrm{R}-\mathrm{O}-\underline{\mathrm{C}}=\mathrm{O}$ & 0.5 & 1.0 & 0.6 & 1.2 & 0.0 & 1.5 & 2.9 & 0.4 \\
\hline$\% \mathrm{C}$ in $\mathrm{O}=\mathrm{C}-\mathrm{O}-\mathrm{C}=\mathrm{O}$ & 0.9 & 1.2 & 1.4 & 0.0 & 3.9 & 0.3 & 0.0 & 0.0 \\
\hline$\% C$ in $-\underline{C}-(C=O)-R$ & 0.0 & 0.0 & 0.0 & 0.0 & 21.4 & 0.0 & 31.1 & 20.7 \\
\hline
\end{tabular}

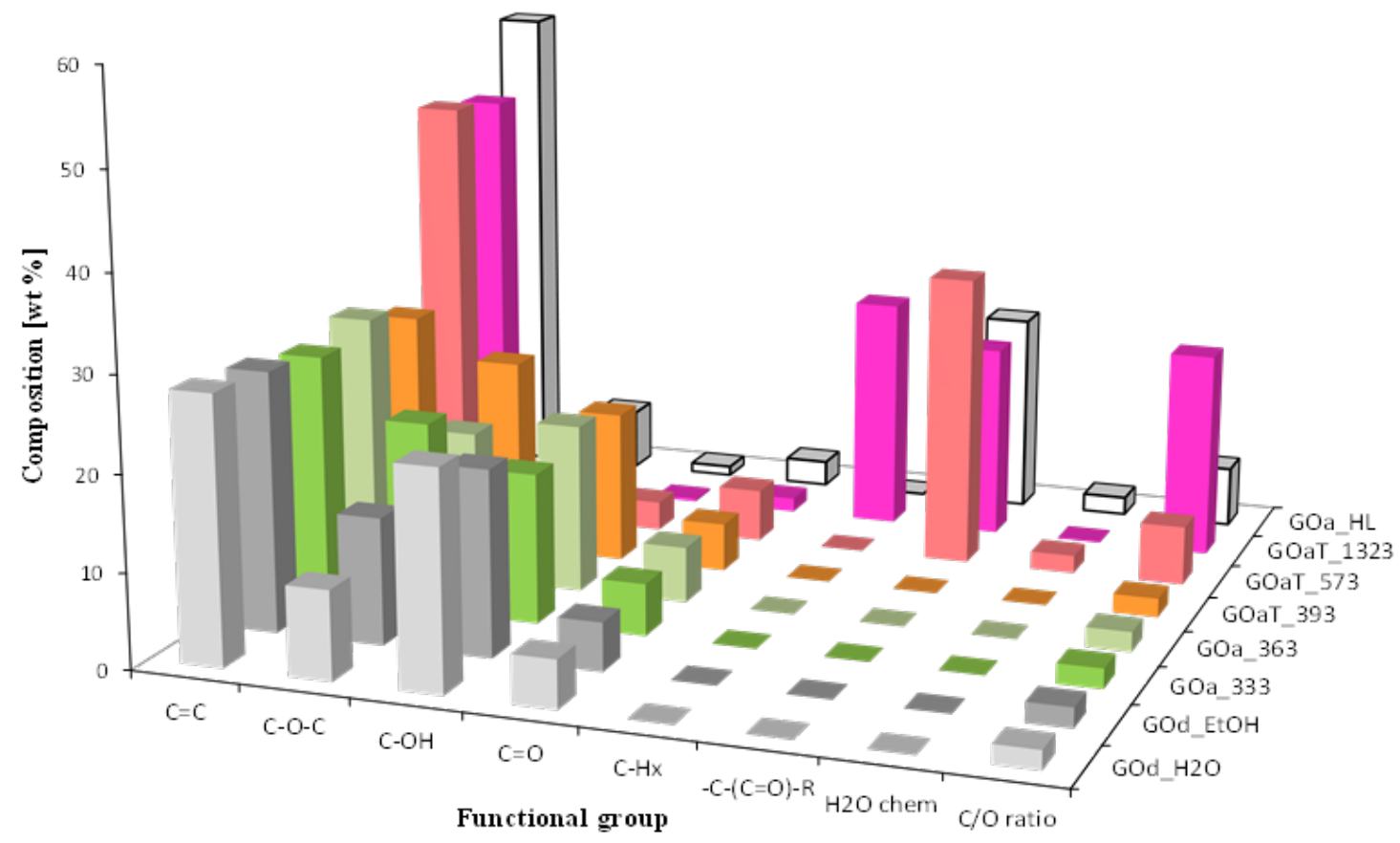

Figure 2. Graphical representation of the XPS data (Table 1) measured for each studied sample. 
XPS data was contrasted with information obtained from the ATR spectra shown in Figure 3. The spectra of GOd_H $\mathrm{H}_{2} \mathrm{O}$ and GOd_EtOH samples were characterized by the several distinct vibrational modes of oxygen functionalities already found in the XPS analysis, i.e., hydroxyls (C-OH at 3000-3750 $\mathrm{cm}^{-1}$, not shown in the figure, and 1222 $\mathrm{cm}^{-1}$ ), carbonyls $\left(>\mathrm{C}=\mathrm{O}\right.$ at $1730 \mathrm{~cm}^{-1}$ ), skeletal vibrations of $\mathrm{sp}^{2}$ hybridized $\mathrm{C}=\mathrm{C}$ from non-oxidized graphite domains at $1620 \mathrm{~cm}^{-1}$ and epoxides (C-O-C at 857, 1024 and $\left.1378 \mathrm{~cm}^{-1}\right){ }^{[32]}$

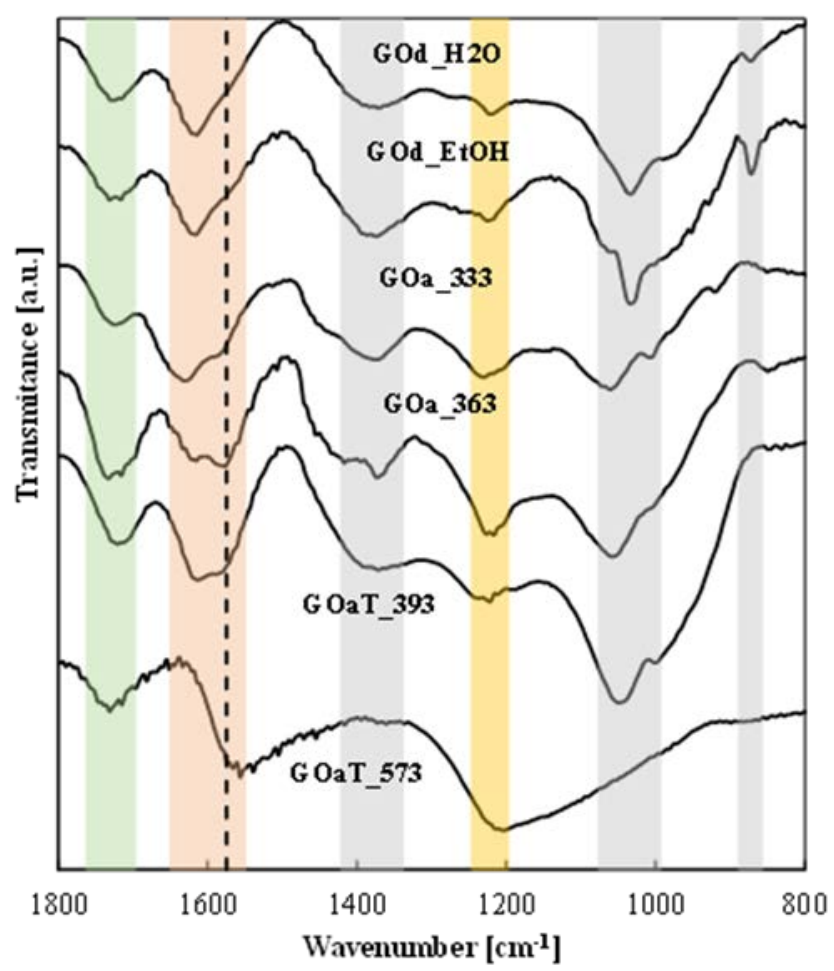

Figure 3. ATR-FTIR main bands for the raw and treated samples.

The GO flakes description inferred from XPS and infrared characterization matches the widely accepted GO model developed by A. Lerf et al.., ${ }^{[3]}$ in which GO is described as constituted by nonstoichiometric platelets wherein both the carbon plane and the edges are mainly decorated with hydroxyl and epoxy functional groups. Carbonyl groups are also present in low quantities, mostly as carboxyl and keto groups at the periphery of the flakes, but also as organic carbonyl defects within the sheets. ${ }^{[34]}$ It should be taken 
into account that the flakes in this study have a huge aspect ratio (lateral dimension / width ratio of $c a$. 30000) in comparison with those normally used in the literature of only some nanometers to few microns of lateral size. For such a high aspect ratio flakes, the contribution of the edges to the total available surface could be considered as negligible, as it was the presence of carboxylic acid groups.

\section{Gelling and drying mechanism in $\mathrm{scCO}_{2}$}

In this work, alcogels are obtained by self-assembly of GO sheets and not by the conventional sol-gel method. Self-assembly is induced by the addition of $\mathrm{CO}_{2}$, an acid gas, which would cause acidification of the ethanol suspension. The decrease on the $\mathrm{pH}$ is described to weaken the electrostatic repulsion between sheets, increasing the number of contact points, thus allowing sheets self-assembly and gel formation.

Examples of gelation by self-assembly of sols of anisotropic sheets induced by $\mathrm{scCO}_{2}$ addition have not been found in the literature. Straight sol-gel gelling induced by compressed $\mathrm{CO}_{2}$ has only been described for polymeric aerogels involving highly

reactive monomers, such as silsesquioxanes, ${ }^{[35]}$ and some biopolymers. ${ }^{[36-39]}$

In the developed supercritical $\mathrm{CO}_{2}$ procedure, the mechanism of gelling and drying was visually followed through the sapphire windows of the high pressure reactor, which allow the acquisition of optical pictures (Figure 4). 


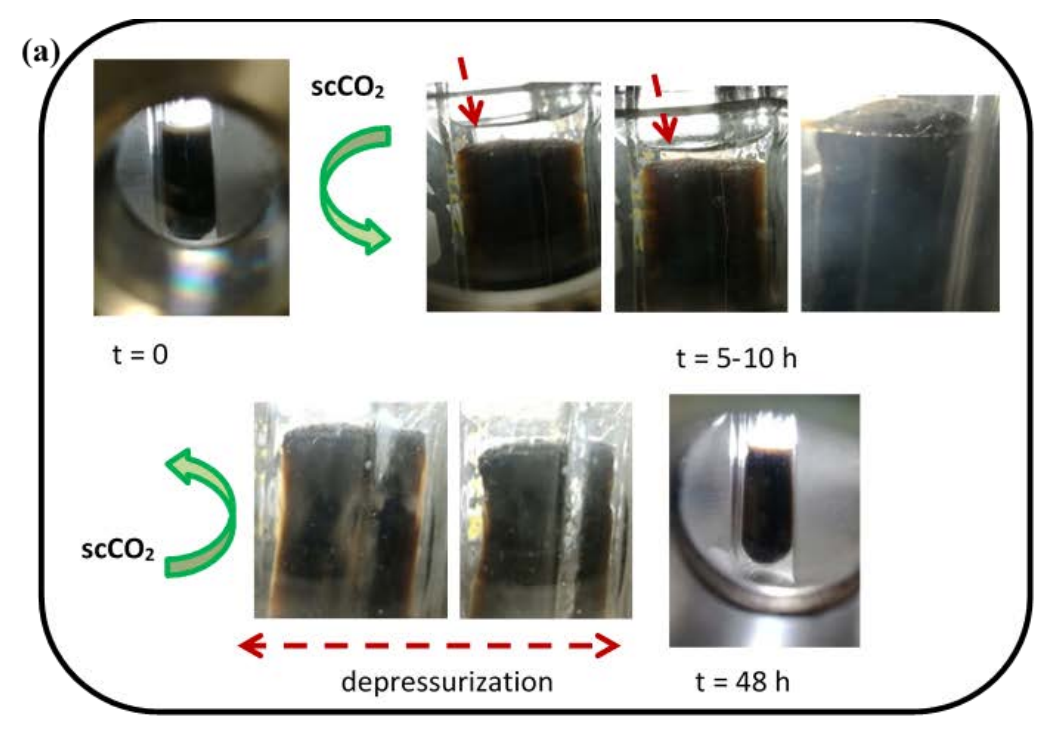

(b)

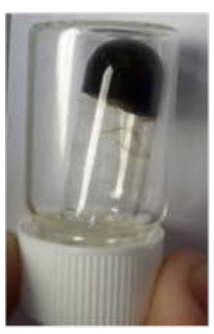

(c)

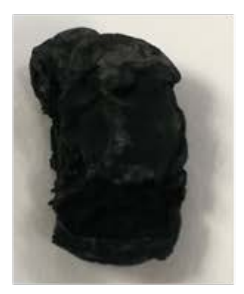

Figure 4. (a) Visual follow-up of aerogel formation through the reactor sapphire windows by taking pictures at different reaction times in a typical standard preparation run carried out in $\mathrm{scCO}_{2}$ at $333 \mathrm{~K}$ and $20 \mathrm{MPa}$, (b) intermediate gel, and (c) dry monolith

Before $\mathrm{CO}_{2}$ addition, the concave shape of the ethanol dispersion meniscus was observed in the vial (Figure 4a at $\mathrm{t}=0$ ). The mechanism of aerogel formation after scCO 2 addition involved two-way mass transfer of $\mathrm{CO}_{2}$ and ethanol to and from the interparticle voids stablished in the GO dispersion. First, the bulk $\mathrm{CO}_{2}$ phase in the 100 $\mathrm{mL}$ reactor diffused into the $1 \mathrm{~mL}$ alcoholic suspension loaded in the vial. By dissolving $\mathrm{CO}_{2}$, the ethanol phase became an expanded liquid, visibly occupying more volume than initially. Concomitantly, the ethanol diffused into the $\mathrm{scCO}_{2}$ phase to equalize concentrations, and the liquid level slowly diminished again. This behavior of the liquid phase is shown in Figure 4a for $t=5-10$ h. In the low density expanded liquid, the flexible GO sheets can easily suffer partial overlapping resulting in the formation of physical cross-linking sites, as occurs in the hydrothermally produced gels. ${ }^{[40]}$

The large number of functional groups in the highly oxidized GO flakes used in this work would end in strong chemical interactions at contact points, thus stabilizing 
the system as an alcogel. After a period of $c a .5 \mathrm{~h}$, the sample in the vial was gelled and the formation of the alcogel can be confirmed by simple vial inversion after depressurization (Figure 4b). The gel recovered from the reactor at this point retained some stability at $279 \mathrm{~K}$ during some weeks, until it finally collapsed due to ethanol evaporation. The strong surface tension developed by the hydrophilic groups within the randomly orientated sheets in the alcogel prevented the liquid from fast flowing, which helped to avoid pore collapse and gel densification. As a consequence, the formation of a dry stable aerogel under a scCO 2 atmosphere was observed to be a slow process that occurs over the course of minimum one day, although experiments in this work were extended to two days to guaranty success. The formation of a "soufflé" on the top of the alcogel is often observed due to swelling in the unrestricted direction. After some hours, a unique homogeneous fluid phase was observed in the reactor, constituted by mixed $\mathrm{CO}_{2}$ and ethanol above the mixture critical point. Finally, isothermal depressurization at the working temperature led to solvent elimination by changing continuously from supercritical fluid to gas phase, i.e., without crossing the curve of coexistence with the liquid phase. Since there was no receding liquid-vapor meniscus, no surface tension was developed; thus, capillary action followed of pore collapse was circumvented. Nevertheless, during depressurization certain shrinkage of the aerogel was observed (Figure 4a depressurization). A dry monolith was recovered from the vial (Figure 4c).

XPS measurements showed that the use of $\mathrm{scCO}_{2}$ technology at $333 \mathrm{~K}$ did not cause any significant reduction in the composition of the GO flakes (Figure 2 and Table 1). The measured $\mathrm{C} / \mathrm{O}$ ratios for the aerogel sample GOa_333 and for the starting GOd_EtOH were in both cases of 2.1. However, a certain redistribution of the functional groups could be discerned. Comparing with the initial GO dispersion in ethanol, the percentage of epoxy groups increases (from ca. 10 to $20 \mathrm{wt} \%$ ) at the 
expense of reducing $\mathrm{OH}$ functionality (from $c a$. 20 to $15 \mathrm{wt} \%$ ). It is assumed that in the gel most of the flakes are interacting through hydrogen bonds. The $\mathrm{scCO}_{2}$ fluid seems to act as a soft desiccant solvent, reducing the number of hydrogen bonds by prompting the cross-linking of contacting GO sheets through the formation of $\mathrm{C}-\mathrm{O}-\mathrm{C}$ linkages. Corroborating this observation, a new band in the ATR spectrum at $1575 \mathrm{~cm}^{-1}$ is clearly visible for the processed aerogel GOa_333 (Figure 3), attributed to $\mathrm{C}=\mathrm{C}$ stretches in the carbon network. This band indicates an increase in the percentage of aromatic regions. The qualitative reduction previously described for graphite oxide triggered by $\mathrm{scCO}_{2}$ at low temperature was not observed in this work. ${ }^{[41,42]}$ Results disparity may be ascribed to differences in the oxygenated groups of the raw materials or to other experimental parameters, like the use in the literature of a continuous flow of $\mathrm{scCO}_{2}$. Quantified substantial conversion of $\mathrm{GO}$ to $\mathrm{rGO}$ in $\mathrm{scCO}_{2}$ has only been reported at temperatures higher than $473 \mathrm{~K} .^{[43]}$

The influence of temperature was analyzed by increasing this parameter from $333 \mathrm{~K}$ in the standard protocol to $363 \mathrm{~K}$. ATR-FTIR spectra (Figure 3) and deconvolution of the XPS spectra obtained for GOa_363 sample (Figure 2 and Table 1) indicate a similar composition than that of sample GOa_333. In term of process, the main effect of the temperature increase is an enhancement of the $\mathrm{CO}_{2}$ diffusion rate in ethanol, with a concomitant increase of ethanol vapor pressure. However, these effects are counteracted by a significant decrease of the solubility of $\mathrm{CO}_{2}$ in the liquid phase. As a consequence, monoliths with similar appearance were obtained at both temperatures. Samples obtained at $333 \mathrm{~K}$ were chosen for further analysis.

The large lateral dimension of the used flakes is an essential parameter in the developed protocol. Indeed, in a separate test performed at $333 \mathrm{~K}$ with GO sheets of only 1-5 $\mu \mathrm{m}$ lateral size, prepared via a modified Hummer's method, ${ }^{[44]}$ the addition of 
compressed $\mathrm{CO}_{2}$ led immediately to colloid instability, re-stacking and flocculation of the flakes. Contrarily, the mobility of large GO sheets in solution is strongly limited. ${ }^{[45]}$ Moreover, the mutual restriction of rotational motion in the high aspect ratio material used in this work makes difficult to adjust their orientation parallel to each other, which allows drying without flocculation. For the studied highly anisotropic 2D GO particles, precipitation is kinetically slow comparing to gelation. Finally, with the procedure described in this work, the use of a flow of $\mathrm{scCO}_{2}$ during gelling and drying hinder the preparation of highly porous aerogels. Recovered monoliths have a size ca. 5-10 times lower than expected. Likely, turbulent flow disturbances induce gel shrinkage. Likewise, stirring the $\mathrm{CO}_{2}$ phase caused the collapse of the suspension and the precipitation of a thin film.

\section{Hydrothermal-lyophilization procedure}

A hydrothermally reduced hydrogel was prepared as a foamed monolith control sample by freeze-drying (sample GOa_HL). ${ }^{[28]}$ In this combined procedure (Figure 1), a significant amount of GO functional groups are removed by temperature during hydrothermal treatment, which prompts linkage among flakes and phase separation in the form of a hydrogel. The reduction was reflected in the XPS analysis of the lyophilized product (Table 1, Figure 2 and Figure 3_SI), which displayed a C/O ratio of 6.4, in which the $\mathrm{C}=\mathrm{C}$ percentage increased from $28 \%$ in $\mathrm{GOd} \_\mathrm{H}_{2} \mathrm{O}$ to $52.3 \%$ in GOa_HL; while the C-O H functionality almost vanished. Hence, a partial restoration of the graphene structure is deduced for the data of the GOa_HL sample. For this sample, SEM micrographs show the formation of a porous sponge with interconnected macropores (Figure 5a).

\section{Structural stability of the aerogel GO monolith}


Often, GO monoliths that have not been reduced, i.e., obtained directly by lyophilization in the sol-cryo process, ${ }^{[46]}$ are highly instable. To increase stability, those monoliths are thermally reduced to rGO. Remarkably, GO aerogels obtained in this work were thermally and mechanically stable without any further treatment.

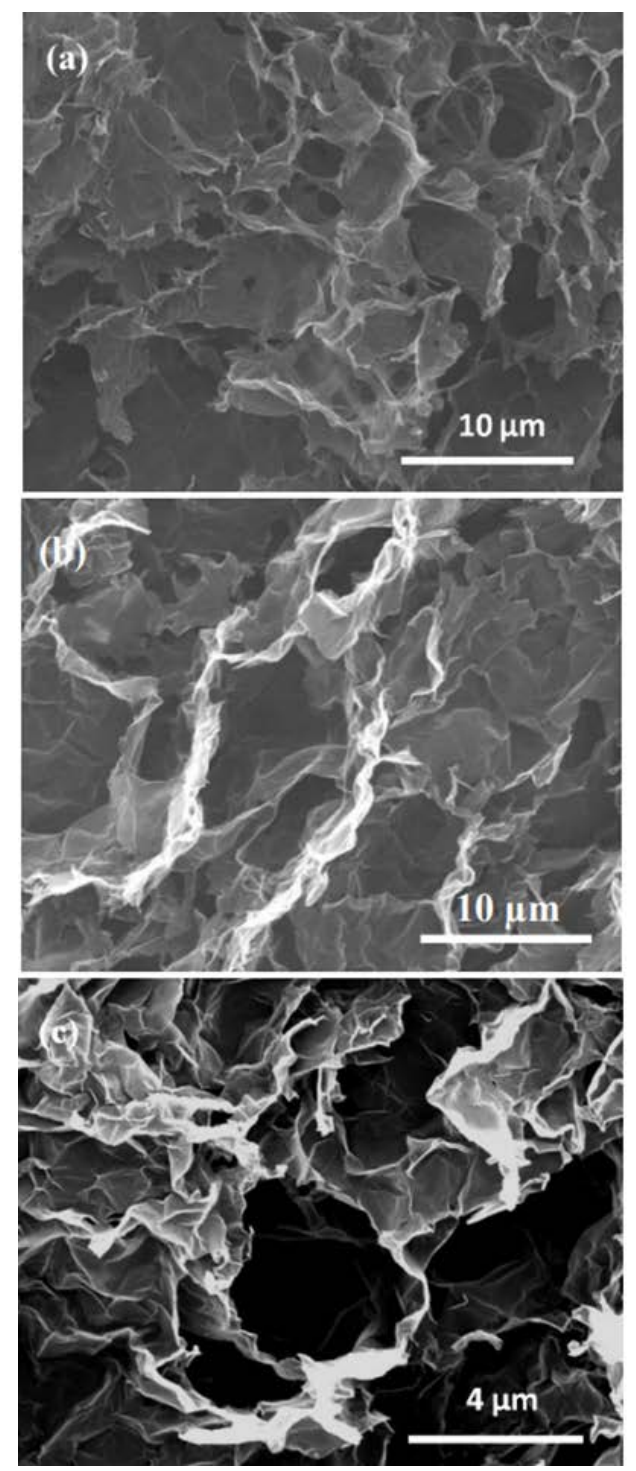

Figure 5. SEM images of: (a) GO sponge produced by a combined hydrothermalfreeze drying conventional technique, and (b,c) monoliths obtained at $333 \mathrm{~K}$ (GOa_333) and 363 K (GOa_363), respectively.

Thermal behaviour of GOa_333 sample was analyzed by TGA under air flow (Figure 6a). The weight loss profile shows first a sharp decay in the 440-500 K 
temperature interval of $c a .30 \mathrm{wt} \%$. This decay is related to desorption and decomposition of the surface oxygenated functional groups in the form of $\mathrm{CO}_{2}$ and $\mathrm{H}_{2} \mathrm{O}$. This value is consistent with the total oxygen percentage of $32 \mathrm{wt} \%$ measured by XPS (Table 1). Afterwards, the decay continues in a more progressive manner in the range of temperature spanning between 500 and $650 \mathrm{~K}$, attributed to loss of intercalated water and elimination of the most stable $\mathrm{C}=\mathrm{O}$ functionalities. Finally, a second sharp decay is observed in the temperature interval $650-700 \mathrm{~K}$, attributed to the pyrolysis of the graphene structure.
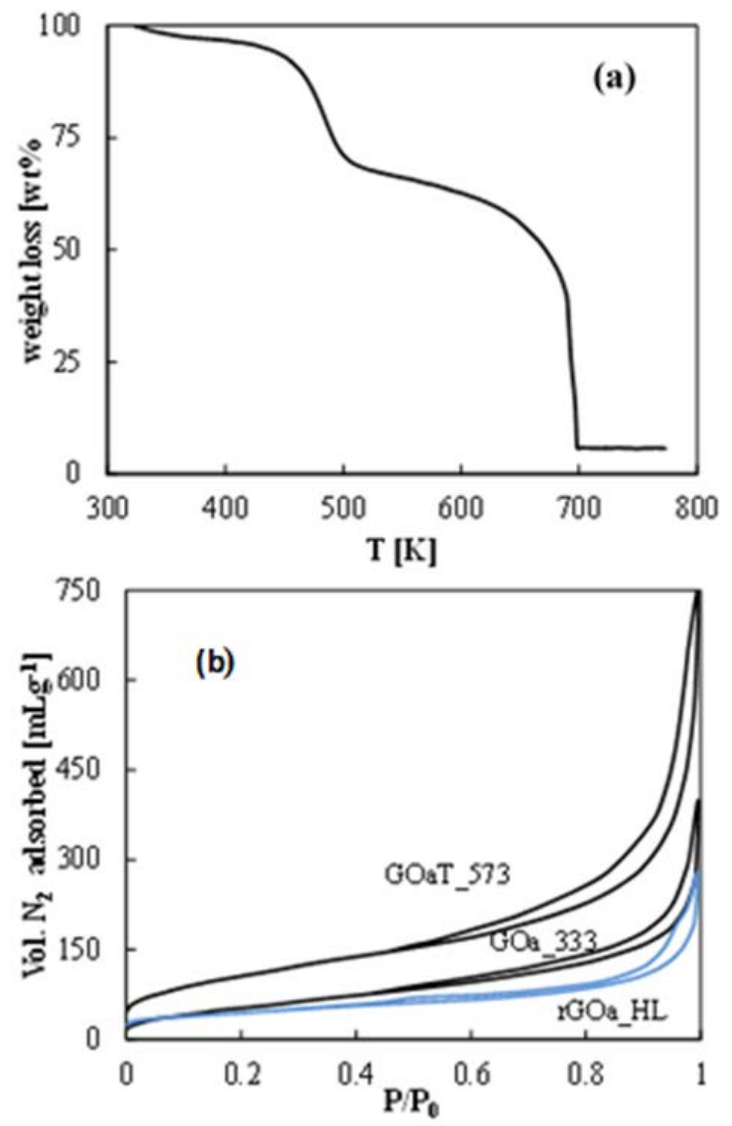

Figure 6. Thermal and textural characterization of different aerogel monoliths: (a) TGA of GOa_333 sample, and (b) $\mathrm{N}_{2}$ adsorption isotherms of GOa_333 as-synthetized and after thermal treatment at $573 \mathrm{~K}$ (GOaT_573), as well as of rGOa_HL sample. 
Based on TGA observations, annealing experiments of GOa_333 monoliths were designed to further understand the thermal characteristics of this compound. Tests were performed under vacuum at 393 and $573 \mathrm{~K}$, giving place to samples GOaT_393 and GOaT_573, respectively. XPS analysis indicated that the sample treated at the lowest temperature did not suffer any modification in regard of GO composition (Figure 2, Table 1). Contrarily, significant thermal reduction occurred at $573 \mathrm{~K}$. Heating at this temperature a compound with a $\mathrm{C} / \mathrm{O}$ ratio of 6.3 was obtained, in which most of the labile epoxy groups were eliminated in favor of the formation of $\mathrm{C}=\mathrm{C} \mathrm{sp}^{2}$ domains, while the contribution of species involving $\mathrm{C}=\mathrm{O}$ did not suffered any significant modification. In fact, the reduction degree achieved for sample GOaT_573 is similar to the one measured for the hydrothermally gelled GOa_HL sample. ATR spectrum also indicates the removal of the bands corresponding to epoxy groups in the GOaT_573 sample, together with an increase in the intensity of the aromatic $\mathrm{C}=\mathrm{C}$ vibration (Figure 3). XPS analysis of the residue recovered after pyrolizing GOa_333 at $1323 \mathrm{~K}$ under Ar flow (sample GOaT_1323) indicated an almost total reduction of the GO flakes, with a C/O ratio of 21.6 (Figure 2 and Table 1).

\section{Gas adsorption and textural properties}

The as-synthetized supercritically prepared monoliths have an initial weight of $c a$. 3.3$3.5 \mathrm{mg}$, which is slightly higher than the initially added GO weight to the vial (ca. 3 $\mathrm{mg}$ ). However, this value is reduced to $c a$. 2.5-2.8 $\mathrm{mg}$ after samples evacuation under vacuum at $393 \mathrm{~K}$. The reduction in weight is due to lose of $\mathrm{CO}_{2}$ adsorbed during synthesis. The density of the monoliths was calculated as the weight of the cylinder after evacuation divided by the geometrical volume. For the supercritically prepared samples, these values were in the order of 7-9 $\times 10^{-3} \mathrm{gcm}^{-3}$, close to the density of air of $1.2 \times 10^{-3} \mathrm{gcm}^{-3}$. The estimated density value for the GOa_HL sample was of $2 \times 10^{-2}$ 
$\mathrm{gcm}^{-3}$, which is one order of magnitude higher than the density measured for GOa_333, indicating a high shrinkage caused by the combined hydrothermal and lyophilization process.

Evacuated monoliths at $393 \mathrm{~K}$ were used to acquired surface area values by low temperature $\mathrm{N}_{2}$ adsorption/ desorption measurements. It is worth mentioning that these measurements for the supercritically prepared products were performed using three monoliths from the same batch, in order to achieve a minimum weight of 7-8 $\mathrm{mg}$, thus reducing specific textural data inaccuracy. Foamed GO samples exhibited a type IV isotherm according to the IUPAC classification, characteristic of mesoporous materials (Figure 6b). Isotherms have a H3-type hysteresis loop related to slit-shaped pores and aggregates of plate-like particles, representative of the studied GO system. This category of isotherms did not reached a plateau at saturation pressure, instead they are extended indefinitely at $\mathrm{P} / \mathrm{P}_{0}=0.99$, representing unrestricted multilayer adsorption in large mesopores and macropores, which makes difficult to give reliable values of pore volume. $^{[47]}$ Therefore, in this work the BET equation was only used to estimate the surface area, giving values in the order of $200-225 \mathrm{~m}^{2} \mathrm{~g}^{-1}$ for GOa_333. The pore volume was roughly estimated by subtracting from the cylinder geometrical volume the volume occupied by the graphene sheets (graphene weight/graphene density reported as $\left.1.06 \mathrm{gcm}^{-3}\right){ }^{[48]}$ For the supercritically prepared samples, this calculation leads to specific empty volume values of $0.65-0.70 \mathrm{~cm}^{3} \mathrm{~g}^{-1}$. The surface area and empty volume values of the GOa_HL sample are $159 \mathrm{~m}^{2} \mathrm{~g}^{-1}$ and $0.50 \mathrm{~cm}^{3} \mathrm{~g}^{-1}$, respectively, slightly lower than those measured for GOa_333 (Figure 6b).

A notable effect of thermal reduction at $573 \mathrm{~K}$ was a substantial increase in the monolith surface area, from values of 207 to $384 \mathrm{~m}^{2} \mathrm{~g}^{-1}$. As it can be observed in Figure $6 \mathrm{~b}$, the surface area increase was mainly generated on the micropore area, likely due to 
the creation of micropores during heating and aerogel setting. Geometrically, the micropores have the highest contribution to the surface area; therefore, even a small increase in the total volume could be accompanied of a large increase in the surface area when the increase is in the micropore volume.

\subsection{Microstructure}

Details of the microstructure of the graphene-based aerogels were revealed by SEM analysis. Samples obtained following the supercritical standard procedure showed a sponge-like porous structure of multiple exfoliated GO sheets (Figure 5b,c).

The structural integrity of the GOa_333 aerogel and the respective reduced counterpart at $573 \mathrm{~K}$ (sample GOaT_573) were mechanically evaluated by static and dynamic mechanical tests.
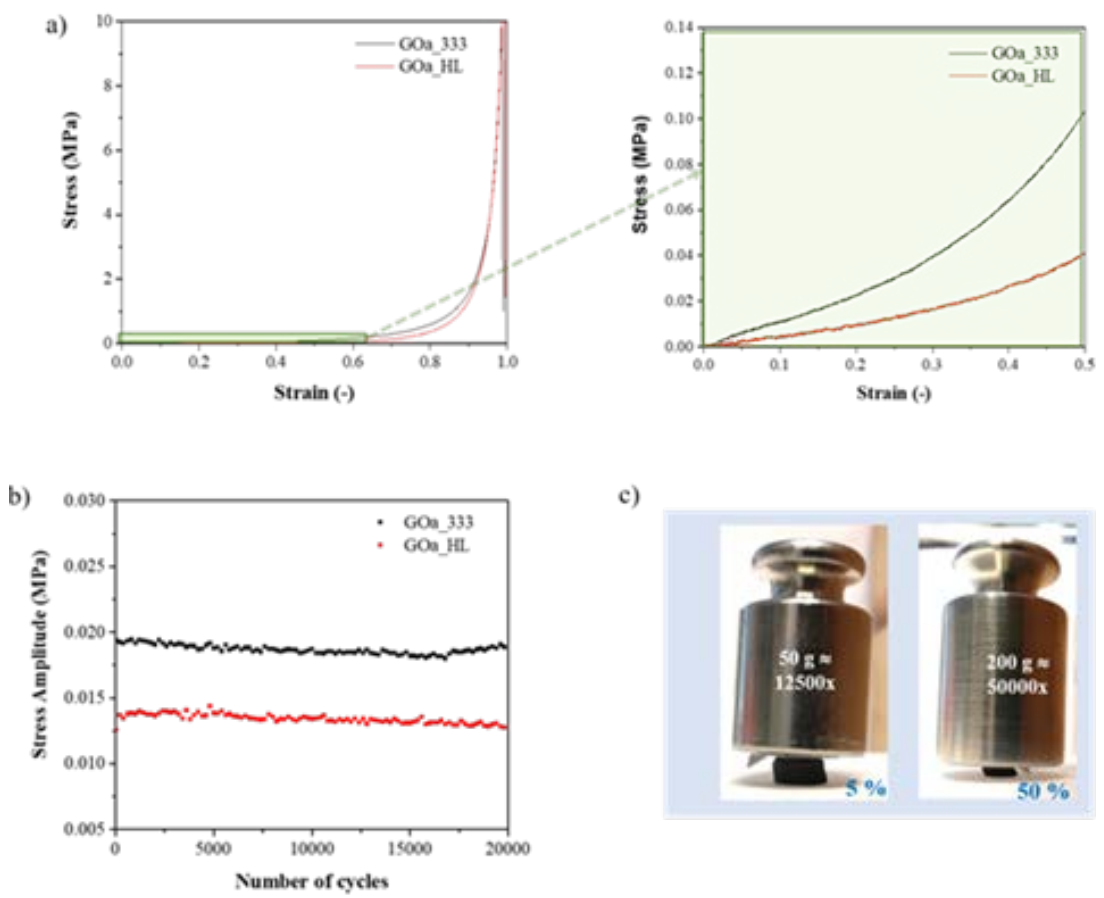

c)

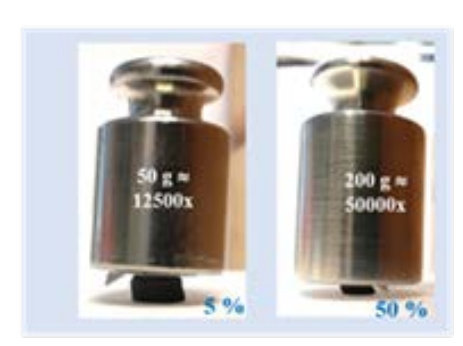

Figure 7. Mechanical tests performed on GOa_333 and reduced GOaT_573 samples: (a) quasi-static uniaxial compression , (b) fatigue at $0.3 \mathrm{~Hz}$ and $10 \%$ of deformation, and (c) demonstration of GOa_333 monoliths elasticity applying different weight: $50 \mathrm{~g}$ 
(12500 times the weight of the monolith), and $200 \mathrm{~g}$ (50000 times the weight of the monolith).

Figure 7a shows the stress-strain curve obtained for the GO-based aerogels. The stressstrain profile curve of the quasi-static uniaxial compression test presents two main regions, elastic and yield. The elastic region is characterized by linear behavior promoted by reversible wall cell bending. In this region, when the load is removed, the material can fully recover the geometrical dimensions. The slope of this linear zone corresponds to the compressive modulus. In this case, the elastic region is up to $20 \%$ of strain and the compressive modulus was calculated for the first $10 \%$ of strain. In the yield region, the pores in the aerogels gradually collapse when stress is slowly increased. After, with continuous loading, the cell walls start to touch each other's and the densification occurs, until the material is fully compressed. This stage is characterized by an abruptly increase in stress. ${ }^{[49,50]}$ The mechanical properties of the aerogels are dependent of several parameters, such as morphology, size and number of pores and degree of interaction between the constituents flakes. Comparing the mechanical performance of pristine GOa_333 and thermally reduced GOaT_573 aerogels, the later showed significantly lower mechanical performance including lower strength and compressive modulus, with values of $111 \pm 4.2$ and $46.5 \pm 2.1 \mathrm{kPa}$, respectively. Indeed, the high density of oxygen functional groups on GOa_333 aerogels allows the establishment of strong interactions between the nanosheets resulting in improved mechanical properties. With the thermal annealing treatment of GOa_333, the oxygen groups were removed in some extent from nanosheets surface, reducing in this way the anchorage sites between the neighboring nanosheets, which promotes the collapse of the structure of the aerogels at lower applied loads. The value of the compressive modulus for the GOa_333 sample was $111 \pm 4.2 \mathrm{kPa}$, which in 
comparison with reported values is in the spot of the higher ranking values. Indeed, it has been reported for graphene aerogels prepared by freeze-casting that compressive modulus values can range from 0.5 to $382 \mathrm{kPa}$, depending on its density. ${ }^{[51,52]}$ In GO aerogels, most of the research has been dedicated to the development of foams with binary systems that consist in GO/crosslinker agents, such as $\mathrm{PVA}^{[50]}$ and PDMS, ${ }^{[53]}$ showing those composites higher compressive modulus values (51 and $290 \mathrm{kPa}$, respectively). In case of polyethylenimine chemically crosslinked GO aerogels, with high specific surface area of ca. $690 \mathrm{~m}^{2} \mathrm{~g}^{-1}$ and low density of $5 \times 10^{-2} \mathrm{gcm}^{-3}$, an outstanding mechanical performance was reported, up to $20 \mathrm{MPa}$ Young's modulus. ${ }^{[6]}$

In order to further evaluate the structural integrity of GO aerogels, fatigue test with compressive strain of $10 \%$ for 20000 cycles where performed on GOa_333 and GOaT_573 samples (Figure 7b). Concerning the fatigue behavior, both aerogels maintain its mechanical integrity, even after 20000 cycles. The stress value necessary to promote $10 \%$ of deformation in the aerogels, at a frequency of $0.3 \mathrm{~Hz}$, remains constant during the cycling compressive tests in values of 19 and $13 \mathrm{kPa}$ for GOa_333 and GOaT_573, respectively. However, for high values of deformation some degradation of the mechanical properties over the time was observed for both aerogels (50\% of $\begin{array}{lllll}\text { deformation, } & 0.3 & \mathrm{~Hz} & \text { and } & 20000\end{array}$

The aerogels were compressible and elastic to some extent, which was surveyed by a simple text performed in the solids obtained at $333 \mathrm{~K}$ (Figure 7c). The size of the monolith was reduced in only $5 \%$ by applying static pressure with a weight of $50 \mathrm{~g}$ (12500 times the weight of the monolith), but it mostly recovered the initial shape after releasing this pressure. Thus, the inherent flexibility of graphene sheets was translated to the 3D macrostructure. However, the monolith was heavily shrunken (ca. $50 \%$ ) after applying a weight of $200 \mathrm{~g}$ (50000 times the weight of the monolith), and the size was 
not recovered by eliminating the weight. This behavior was likely due to partial breakage of the interior network of the monolith under compression.

\section{Conclusions}

Monoliths of GO aerogels were prepared in a low-temperature single-step procedure using exclusively $\mathrm{scCO}_{2}$ technology, circumventing the standard synthesis protocols involving high-temperature hydrothermal sol-gel chemistry, lyophilization and/or drying at the critical point of ethanol. Although many methods have been described for the synthesis of reduced rGO aerogels, the novelty of the reported $\mathrm{scCO}_{2}$ method is that highly oxygenated GO aerogels are produced, while using a simple, fast, and economical method. Rigid and stable three-dimensional GO networks were obtained by direct gelling of the high aspect ratio sheets constituting the GO precursor dispersed in ethanol and their self-assembly assisted by $\mathrm{scCO}_{2}$ addition. The resulting GO aerogels show light weight and low density (7-9 x 10 gcm $\left.^{-3}\right)$, large BET surface area (200-225 $\mathrm{m}^{2} \mathrm{~g}^{-1}$ ) and high thermal stability (up to $500 \mathrm{~K}$ in air). The mechanical study shows that the GO monoliths support more than 12000 times their own weight. A large number of applications can be envisaged for GO networks before reduction, since they combine the textural advantages of graphene with convenient hydrophilicity and attractive surface chemistry given by the oxygen functional groups decorating the $\mathrm{sp}^{2}$ basal planes. The usability of this remarkable material is thus enhanced by translating the key properties of individual GO building blocks into macroscopic 3D architectures.

\section{Experimental}

\section{Materials}


GO sheets (ca. $30 \mu \mathrm{m}$ lateral dimensions) were purchased from Graphenea Inc. (Spain), supplied as a dispersion in water with a concentration of $4 \mathrm{mgmL}^{-1}$. Compressed $\mathrm{CO}_{2}$ (99.95 wt\%) was supplied by Carburos Metálicos S.A. Absolute ethanol $(\mathrm{EtOH})$ was obtained from Scharlau Chemie S.A.

\section{Equipment and Methods}

The synthetic procedure starts by exchanging the water in the commercial GO dispersion (sample GOd_ $\mathrm{H}_{2} \mathrm{O}$ ) by ethanol. Water replacement was performed in successive steps of centrifugation (11000 rpm) / sonication (30 min) by using ethanol:water mixtures with increased alcohol concentration, 30:70, 50:50, 70:30 and finally 100:0. It is worth mentioning that sonication buildup a tensile load that likely

triggers partial breakage of the sheets. ${ }^{[27]}$ A stable colloidal suspension of GO in EtOH with a concentration of $c a .3 \mathrm{mgmL}^{-1}$ was thus obtained (sample GOd_EtOH). Aerogel monoliths were prepared in small assay tubes of $2 \mathrm{~mL}$ loaded with $1 \mathrm{~mL}$ of GOd_EtOH dispersion. In a standard experiment, three of these vials were placed into a non-stirred high pressure reactor of $100 \mathrm{~mL}$ volume (TharDesign) with two opposite sapphire windows. Liquid $\mathrm{CO}_{2}$ was flushed into the vessel to pressurize the system at $6 \mathrm{MPa}$. The vessel was gently heated at either 333 or $363 \mathrm{~K}$ and then pressurized up to $20 \mathrm{MPa}$. These conditions were kept for a period of 2 days. Pressure decrease to ambient conditions was achieved by slow release of $\mathrm{CO}_{2}$. The depressurization was performed under isothermal condition to avoid entering the two-phase region for the compressed $\mathrm{CO}_{2}$, which would induce pore collapse in the structure. GO aerogel samples were isolated as black cylindrical monoliths of $c a .3 \mathrm{mg}$, and termed as GOa_333 and GOa_363 indicating the processing temperature. Other studied experimental parameters were the lateral dimensions of GO sheets (30 and $5 \mu \mathrm{m}$ ), stirring ( 0 and $250 \mathrm{rpm}$ ) and flow of $\mathrm{CO}_{2}\left(0\right.$ and $\left.2 \mathrm{mLmin}^{-1}\right)$. 
For comparison, a 3D GO network was synthetized by a conventional reported method, ${ }^{[28]}$ consisting of the hydrothermal reduction at $453 \mathrm{~K}$ and for $12 \mathrm{~h}$ of a similar Graphenea aqueous suspension. In a second step, the recovered hydrogel is lyophilized. The obtained sample was termed GOa_HL.

\section{Characterization}

To determine the extent of oxidation of the GO sheets, X-ray photoelectric spectroscopy (XPS) was recorded for C and O in a XPS Spectrometer Kratos AXIS Supra, using Al-Ka $(20 \mathrm{eV})$ radiation emitted at $225 \mathrm{~W}(15 \mathrm{~mA} / 15 \mathrm{kV})$. The binding energies of the resulting spectra were corrected employing the binding energy of adventitious carbon $(284.6 \mathrm{eV})$ in the $\mathrm{C} 1 \mathrm{~s}$ region. The backgrounds were corrected using Shirley baselines. All the analyzed regions were deconvoluted by means of mixed Gaussian-Lorentzian functions (90:10). The quantitative analyses were based on the atomic sensitivity factors stored in the CasaXPS database (v2.3.12Dev6). In the supplementary information (SI), the C 1s and O 1s core level spectra deconvolution is shown for each studied sample, as well as the criteria for band assignment (Figure 14_SI). The functional groups were also investigated by means of reflectance attenuate mode Fourier transformed infrared (ATR-FTIR) spectroscopy (Nicolet Thermo Scientific apparatus). The textural properties were determined by $\mathrm{N}_{2}$ adsorption/ desorption at $77 \mathrm{~K}$ using an ASAP 2000 Micromeritics Inc. Samples were first degassed at $393 \mathrm{~K}$ for $24 \mathrm{~h}$. The surface area $\left(\mathrm{S}_{\mathrm{a}}\right)$ was calculated applying the BET equation. Morphological features were examined by scanning electron microscopy (SEM, Quanta FEI 200). The thermal stability was analyzed in air flow by thermogravimetric analysis (TGA, Netzsch-STA 449 F1 Jupiter) using a temperature increase step of $5 \mathrm{Kmin}^{-1}$. The mechanical behavior of selected samples was evaluated by quasi-static compression tests at a cross-head speed of $0.5 \mathrm{mms}^{-1}$ and cyclic stability by fatigue tests at a 
frequency of $0.3 \mathrm{~Hz}$ and $10 \%$ of deformation. Both tests were conducted using Microservo Magnetic Tester equipment (MMT-101N Shimadzu Corporation).

\section{Acknowledgments}

This work was financed by the Spanish National Plan of Research with projects CTQ2014-56324, CTQ2017-83632 and MAT2017-86616-R. C.D., G.T, A.B, G.G., S.S., J.F. and A.L.P. acknowledge the financial support from the Spanish MEC, through the Severo Ochoa Program for Centers of Excellence in R\&D (SEV-2015-0496). This work (A.B.) has been done in the framework of the doctoral program "Chemistry" of the Universitat Autònoma de

Barcelona.

A.L.P. acknowledges the RyC-2012-11588 contract. A.B. acknowledges FPI grant. G.G. acknowledges the funding by EC H2020 under individual fellowship Marie Sklodowska-Curie (NANOTER, Grant Agreement 708351). P.M. and S.P. acknowledge the FCT/MCTES for respectively, a research contract under the Program Investigator (IF/00917/2013/ CP1162/CT0016) and PhD grant SFRH/BD/111515/2015; also UID/EMS/00481/2013-FCT and CENTRO-01-0145-FEDER-022083.

Keywords: graphene oxide $\bullet$ aerogel $\bullet$ supercritical $\mathrm{CO}_{2} \cdot$ mesoporous $\bullet$ mechanical integrity

\section{References}

[1] H. Wang, X. Yuan, G. Zeng, Y. Wu, Y. Liu, Q. Jiang, S. Gu, Adv. Colloid Interf. Sci. 2015, 221, 41-59.

[2] D.R. Dreyworsleer, S. Park, C.W. Bielawski, R.S. Ruoff, Chem. Soc. Rev. 2010, 39, 228-240.

[3] E. García-Bordejé, S. Víctor-Román, O. Sanahuja-Parejo, A. M. Benito, W. K. Maser, Nanoscale 2018, 10, 3526-3539. 
[4] S. Pei, H-M. Cheng, Carbon 2012, 50, 3210-3228.

[5] T. Ma, P.R. Chang, P. Zheng, F. Zhao, X. Ma, Mater. Chem. Phys. 2014, 146, 446451.

[6] M.B. Lim, M. Hu, S. Manandhar, A. Sakshaug, A. Strong, L. Riley, P.J. Pauzauskie, Carbon 2015, 95, 616-624.

[7] C. Cheng, D. Li, Adv. Mater. 2013, 25, 13-30.

[8] J. Wang, X. Gao, Y. Wang, C. Gao, RSC Adv. 2014, 4, 57476-57482.

[9] V. Chabot, D. Higgins, A. Yu, X. Xiao, Z. Chena, J. Zhang, Energy Environ. Sci. 2014, 7, 1564-1596.

[10]Q. Fang, Y. Shen, B. Chen, Chem. Eng. J. 2015, 264, 753-771.

[11]F. Perrozzi, S. Prezioso, J. Phys.: Condens. Matter. 2015, 27, 013002.

[12] F. Liu, S. Chung, G. Oh, T.S. Seo, ACS Appl. Mater. Interf. 2012, 4, 922-927.

[13]R. Yu, Y. Shi, D. Yang, Y. Liu, J. Qu, Z.Z. Yu. ACS Appl. Mater. Interf. 2017, 9, 21809-21819.

[14]Q. Fang, B. Chen. J. Mater. Chem. A 2014, 2, 8941-8951.

[15]N. Kostoglou, V. Tzitzios, A.G. Kontos, K. Giannakopoulos, C. Tampaxis, A. Papavasiliou, G. Charalambopoulou, T. Steriotis, Y. Li, K. Liao, K. Polychronopoulou, C. Mitterer, C. Rebholz, Int. J. Hydr. Ene. 2015, 40, 6844-6852.

[16]M. Seo, D. Yoon, K.S. Hwang, J.W. Kang, J. Kim, Carbon 2013, 64, 207-218.

[17]A. Alazmi, O. El Tall, S. Rasul, M.N. Hedhili, S. P. Patole, P.M.F.J. Costa, Nanoscale 2016, 8, 17782-17787.

[18]L. Hrubesh, Chem. Ind. 1990, 24, 824-827

[19]Y. Cheng, S. Zhou, P. Hu, G. Zhao, Y. Li, X. Zhang, W. Han, Scientific Reports 2017, 7, 1439.

[20] M.J. Van Bommel, A.B. de Haan, J. Non-Cryst. Solids 1995, 186, 78-82. 
[21] Smirnova, W. Arlt, J. Sol-Gel Sci. Tech. 2003, 28, 175-184.

[22]C. Tan, B.M. Fung, J.K. Newman, C. Vu, Adv. Mater. 2001, 13, 644-646.

[23] S.P. Sasikala, P. Poulin, C. Aymonier, Adv. Mater. 2016, 28, 2663-2691.

[24]M.A. Worsley, T.Y. Olson, J.R. I. Lee, T,M. Willey, M.H. Nielsen, S.K. Roberts, P.J. Pauzauskie, J. Biener, J.H. Satcher, T.F. Baumann, J. Phys. Chem. Lett. 2011, 2, 921-928.

[25]L. Li, J. Xu, G. Li, X. Jia, Y. Li, F. Yang, L. Zhang, C. Xu, J. Gao, Y. Liu, Z. Fang, Chem. Eng. J. 2016, 284, 78-84.

[26]H. Gao, G. Hu, RSC Adv. 2016, 6, 10132-10143.

[27]S. Pan, I.A. Aksay, ACS Nano 2011, 5, 4073-4083.

[28]G. Gonçalves, S.M.G. Pires, M.M.Q. Simoes, M.P.M.S. Neves and P.A.A.P. Marques, Chem. Commun., 2014, 50, 7673-7676.

[29] J. I. Paredes, S. Villar-Rodil, A. Martnez-Alonso, J. M. D. Tascon, Langmuir 2008, 24, 10560-10564.

[30] S.P. Raman, P. Gurikov, I. Smirnova, J. Supercrit. Fluids 2015, 106, 23-33.

[31]E. B. Nursantkongo, A. Nugroho , S.-A. Hong , S. J. Kim , K. Y. Chung , J. Kim, Green Chem. 2011, 13, 2714-2718.

[32] M. Acik, C. Mattevi, C. Gong, G. Lee, K. Cho, M. Chhowalla, Y.J. Chabal, ACS Nano 2010, 4, 5861-5868.

[33]H. He, J. Klinowsky, M. Forster, A. Lerf, Chem. Phys. Lett. 1998, 287, 53-56.

[34]D.R. Dreyer, S. Park, C.W. Bielawski, R.S. Ruoff, Chem. Soc. Rev. 2010, 39, 228240.

[35]D.A. Loy, E.M. Russick, S.A. Yamanaka, B.M. Basugher, K.J. Shea, Chem. Mater. 1997, 9, 2264-2268. 
[36]P. Gurikov, S.P. Raman, D. Weinrich, M. Ficke, I. Smirnova, RSC Adv. 2015, 5, 7812-7818.

[37] M.L. Floren, S. Spilimbergo, A. Motta, C. MIgliaresi, Biomacromolec. 2012, 13, 2060-2072.

[38] S.P. Raman, P. Gurikov, I. Smirnova, J. Supercrit. Fluids 2015, 106, 23-33.

[39] M. Martins, A.A. Barros, S. Quraishi, P. Gurikov, S.P. Raman, I. Smirnova, A.R.C. Duarte, R.L. Reis, J. Supercrit. Fluids 2015, 106, 152-159.

[40]Y. Xu, K. Sheng, C. Li, G. Shi, ACS Nano 2010, 7, 4324-4330.

[41] M. Sarno, L. Baldino, C. Scudieri, S. Cardea, P. Ciambelli, E. Reverchon, J. Supercrit. Fluids 2016, 118, 119-127.

[42]L. Baldino, M. Sarno, S. Cardea, S. Irusta, P. Ciambelli, J. Santamaria, E. Reverchon, Ind. Eng. Chem. Res. 2015, 54, 8147-8156.

[43]C.Y. Kong, W-L. Song, M.J. Meziani, K.N. Tackett, L. Cao, A.J. Farr, A. Anderson, Y-P. Sun, J. Supercrit. Fluids 2012, 61, 206-2011.

[44]D. Kepić, S. Sandoval, A. Pérez del Pino, E. György, L. Cabana, B. Ballesteros, G. Tobias, Chem. Phys. Chem. 2017, 18, 935-941.

[45]H. Bai, C. Li, X. Wang, G. Shi, J. Phys. Chem. C 2011, 115, 5545-5551.

[46]H. Sun, Z. Xu, C. Gao, Multifunctional, Adv. Mater. 2013, 25, 2554-2560.

[47]K.S.W. Sing, D.H. Everett, R.A.W. Haul, L. Moscou, R.A. Pierotti, J. Rouquerol, T. Siemieniewska, Pure \& Appl. Chem. 1985, 57, 603-619.

[48] M.A. Rafiee, J. Rafiee, Z. Wang, H. Song, Z-Z. Yu, N. Koratkar, ACS Nano 2009, 3, 3884-3890.

[49]Y.-D. Gao, Q.-Q. Kong, Z. Liu, X.-M. Li, C.-M. Chen, R. Cai, RSC Adv. 2016, 6, 9851-9856. 
[50]X. Zhang, Z. Sui, B. Xu, S. Yue, Y. Luo, W. Zhan, B. Liu, J. Mater. Chem. 2011, 21, 6494-6497.

[51]H. Chen, Z. Li, X. Liu, H.-M. Ren, X.-Q. Tang, P. Zhang, Y.-H. Ding, Mater. Res. Express 2017, 4, 25601.

[52]Y. He, Y. Liu, T. Wu, J. Ma, X. Wang, Q. Gong, W. Kong, F. Xing, Y. Liu, J. Gao, J. Hazard. Mater. 2013, 260, 796-805.

[53]P. Samora, C.F. Woellner, T. Li, S. Vinod, S. Ozden, S. Kosolwattana, S. Bhowmick, L.X. Duy, R.V. Salvatierra, B. Wei, S.A.S. Asif, J.M. Tour, R. Vajtai, J. Lou, D.S. Galvão, C.S Tiwary, P.M. Ajayan, Adv. Mater. Interfaces 2017, 4, 1700030. 